\title{
Are Periventricular Lesions Specific for Multiple Sclerosis?
}

\section{Gianna Casini, Mary Yurashevich, Rohini Vanga, Subasini Dash, Suhayl Dhib-Jalbut, Brian Gerhardstein, Matilde Inglese, Win Toe and} Konstantin E Balashov*

Department of Neurology, Robert Wood Johnson Medical School, New Brunswick, New Jersey, USA

\begin{abstract}
Background: The presence of periventricular lesions (PVL) on MRI scans is part of the revised McDonald multiple sclerosis (MS) diagnostic criteria. However, PVL can be found in other neurological diseases including stroke and migraine. Migraine is highly prevalent in patients with MS
\end{abstract}

Objective: To determine if PVL are specific for patients with MS compared to stroke and migraine.

Methods: We studied patients diagnosed with clinically isolated syndrome (CIS), relapsing-remitting MS (RRMS), migraine, and ischemic stroke. The number, location and the volume of PVL were identified on brain MRI scans and analyzed.

Results: The number and volume of PVL adjacent to the body and the posterior horn of the lateral ventricles were significantly increased on fluid-attenuated inversion recovery MRI in RRMS compared to migraine. There were no significant differences in the total number and volume of PVL in ischemic stroke patients compared to the agematched RRMS patients nor in the number and volume of PVL adjacent to the anterior and temporal horns of the lateral ventricles on FLAIR images in migraine compared to CIS or RRMS.

Conclusion: In contrast to PVL adjacent to the body and the posterior horn of the lateral ventricles, PVL adjacent to the anterior and temporal horns of the lateral ventricles may not be specific for CIS/RRMS when compared to migraine, the disease highly prevalent among patients with MS. PVL are not specific for MS when compared to ischemic stroke.

Keywords: Multiple sclerosis; Migraine; Stroke; MRI

Abbreviations: CIS: Clinically Isolated Syndrome; FLAIR: Fluid-attenuated Inversion Recovery; MS: Multiple Sclerosis; PVL: Periventricular Lesions; ROC: Receiver Operating Characteristic; RRMS: Relapsing-Remitting MS; T2WI: T2-weighted Images

\section{Introduction}

The presence of periventricular lesion (PVL) has been considered a hallmark of multiple sclerosis (MS) and was included in the 2010 revised McDonald MS criteria of "dissemination in space" [1] based on observation of Swanton et al. [2]. The original study used a population of young patients presenting with their first clinically isolated syndrome (CIS). However, "The performance of the MRI diagnostic criteria in patients ...with other multifocal diseases of CNS white matter have not been systematically addressed" as was noted by Montalban et al. [3]. PVL were reported in a number of neurological disorders which can mimic MS such as vitamin B12 deficiency [4], migraines [5] and stroke, especially in women [6]. The prevalence of migraine in patients with MS is approximately $25 \%$ [7-9]. In this study, we performed a retrospective analysis of brain MRI and clinical records of patients in order to determine if PVL presence, volume and location are specific for patients with CIS and relapsing-remitting MS (RRMS) as compared to migraine or stroke.

\section{Methods}

\section{Patients}

In this study we identified records of patients who were recently seen in the Department of Neurology at Robert Wood Johnson Medical School and had a clinical diagnosis of CIS as per CHAMPS criteria [10] (without migraine or stroke), clinically-definite RRMS as per Poser criteria [11] (without migraine or stroke), migraine (without MS, CIS or stroke) or ischemic stroke (without CIS, RRMS or migraine)
Additionally, these patients had to have brain MRI available in Digital Imaging and Communications in Medicine (DICOM) format. The study population included patients between the ages of 18 and 60 . Exclusion criteria were: history of neurological disease affecting the CNS other than ischemic stroke, migraine, CIS or clinically-definite RRMS or clinical history or laboratory tests consistent with systemic disease associated with demyelinating lesions in the CNS such as Lyme, vitamin B12 deficiency, sarcoidosis, lupus, syphilis, rheumatoid arthritis or diabetes mellitus. The study was approved by the local IRB. The final patient pool consisted of CIS ( $n=10)$, RRMS ( $n=36)$, migraine $(\mathrm{n}=32)$ and ischemic stroke $(\mathrm{n}=18)$. Their demographic characteristics are depicted in Table 1 (CIS, RRMS and migraine) and Table 3 (stroke).

\begin{tabular}{|c|c|c|c|c|}
\hline Patient Group & \multicolumn{2}{|c|}{$\begin{array}{c}\text { Number of patients } \\
\text { (Male/Female }\end{array}$} & Age, Mean \pm SD & Minimum Age / \\
\hline & \multicolumn{2}{|c|}{ Ratio) } & & \\
\hline & 36 & $(5 / 31)$ & $39.44 \pm 9.81$ & $22 / 58$ \\
\hline RRMS & 10 & $(3 / 7)$ & $34.8 \pm 13.81$ & $19 / 58$ \\
\hline CIS & 32 & $(9 / 23)$ & $39.97 \pm 9.57$ & $22 / 57$ \\
\hline
\end{tabular}

Abbreviations: RRMS = Relapsing-Remitting Multiple Sclerosis, CIS = Clinically Isolated Syndrome

Table 1: Age and Gender Data for RRMS, CIS and Migraine Patient Groups.

*Corresponding author: Konstantin E. Balashov, 125 Paterson Street, 6th Floo New Brunswick, NJ, 08901, USA, Tel: (732) 235-7727; Fax: (732) 906-5705; E-mail: kbalashov@yahoo.com

Received March 15, 2013; Accepted April 27, 2013; Published May 03, 2013

Citation: Casini G, Yurashevich M, Vanga R, Dash S, Dhib-Jalbut S, et al. (2013) Are Periventricular Lesions Specific for Multiple Sclerosis? J Neurol Neurophysio 4: 150. doi:10.4172/2155-9562.1000150

Copyright: $\odot 2013$ Casini G, et al. This is an open-access article distributed unde the terms of the Creative Commons Attribution License, which permits unrestricted use, distribution, and reproduction in any medium, provided the original author and source are credited. 
Citation: Casini G, Yurashevich M, Vanga R, Dash S, Dhib-Jalbut S, et al. (2013) Are Periventricular Lesions Specific for Multiple Sclerosis? J Neurol Neurophysiol 4: 150. doi:10.4172/2155-9562.1000150

\section{Neuroimaging analysis}

We analyzed the lesion number (count) and volume of PVL distributed across four areas of the lateral ventricles: anterior (frontal) horn, the body of the lateral ventricles, posterior (occipital) horn and temporal horn, as defined in Dr. Lawrence B. Stack's, Head CT Scan Interpretation: An Organized Approach to Seeing Inside the Head (2007)_(http://www.accessem.com/login.aspx). Both T2-weighted images (T2WI) and fluid-attenuated inversion recovery (FLAIR) MRI images performed on $1.5 \mathrm{~T}$ MRI machine were analyzed using JIM version 5.0 software (Xinapse systems, UK, http://www.xinapse. $\operatorname{com} /$ ) to compile PVL volumes that spanned multiple axial views of the brain MRI for both FLAIR and T2WI. This manual outlining technique yielded lesion volumes as well as lesion counts for each of the four anatomical areas of the lateral ventricles. Supplemental Figure 1 depicts the example of lesion outline using JIM for axial images. Since conventional MRI cannot differentiate nonspecific frontal capping from small white matter lesions that are a result of multiple sclerosis, periventricular "caps" around the frontal horns and hyperintense outlines of the ventricles were included [12]. PVL were identified if there was no normal-appearing white matter between the lesion and the border of the ventricle. If there was normal-appearing white matter appreciated between two PVL, and it was evident on all axial views depicting those lesions, then the lesions were counted separately [12].

GraphPad Prism software (LaJolla, CA, http://www.graphpad. $\mathrm{com} /$ ) was used for statistical analysis of collected data using unpaired t-tests. Using SYSTAT Software (http://www.systat.com/), we could also determine what lesion volume or count for particular MRI modality (e.g., FLAIR or T2WI) would yield the highest accuracy of a correct differentiation between MS or migraine diagnosis. SYSTAT's two-step approach included its binary logistic regression and receiver operating characteristic (ROC) curves [13] that generated cutoff points, or thresholds, for each of the data sets that were compared (e.g., total PVL volumes of CIS versus migraine) [14]. Once the thresholds were generated, we calculated the corresponding accuracy using ROC's sensitivity and specificity data. The threshold that aligned with the maximal accuracy was then used to create a Model classification table. The Model classification table enabled us to see how many patients were classified correctly or incorrectly as being CIS, RRMS or migraine.

\section{Results}

First, we compared the volume and the number (count) of the PVL in the four anatomical regions of the lateral ventricles in CIS and RRMS versus migraine. (Table 1) depicts our patient groups' data and characteristics. These three groups were predominantly females.

As shown in (Table 2), not all anatomical periventricular areas were equally specific for CIS/RRMS patients as compared to migraine on FLAIR and T2WI.

The maximal difference identified between patient groups was seen when comparing the volume of PVL adjacent to the body of the lateral ventricles and the posterior horn. In those two anatomical areas, the average volume of these PVL was between 1.9 to 6.2 times greater in RRMS patients $(\mathrm{p}<0.033)$ and 3.3 to 8.9 times higher in CIS patients

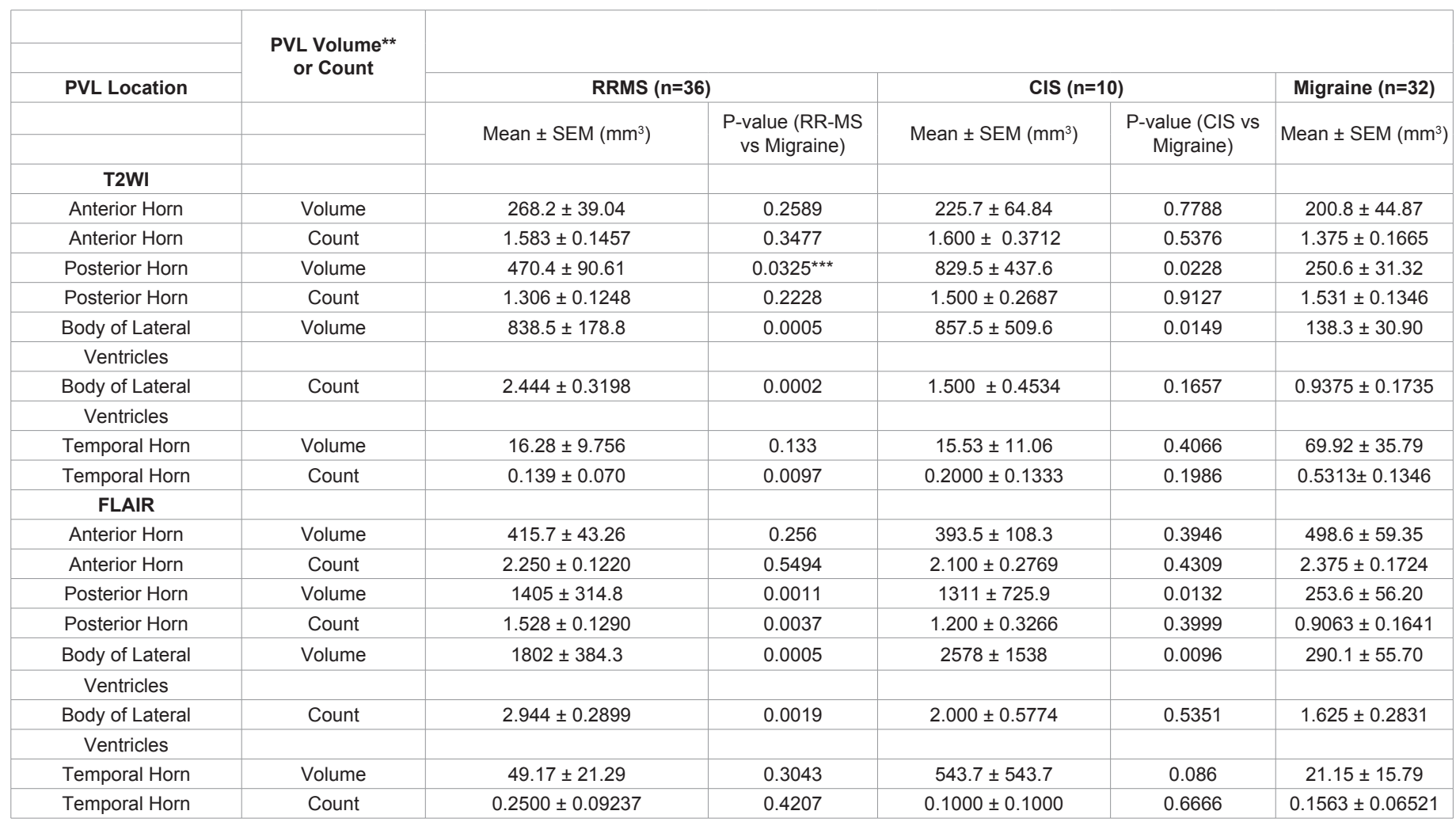

${ }^{*} \mathrm{PVL}=$ Periventricular Lesions

** T2WI and FLAIR images were analyzed using JIM version 5.0 software to measure PVL volume. Unpaired t-test was used for statistical analysis and calculation of p-value.

*** P-values in bold are considered statistically significant.

Table 2: Comparison of $\mathrm{PVL}^{*}$ Volume and Count for Four Anatomical Regions for RRMS and CIS versus Migraine Patients 
$(\mathrm{p}<0.023)$ as compared to migraine for both FLAIR and T2WI. Greater PVL volume difference between CIS/RRMS and migraine was seen using FLAIR sequence as opposed to T2WI. With regard to PVL count, significant statistical difference was identified for RRMS but only for lesions adjacent to the temporal horn and body of the lateral ventricles (as seen on T2WI) and the posterior horn and body of the lateral ventricles (as seen on FLAIR). There were no significant differences between the number and volume of PVL adjacent to the anterior horns of the lateral ventricles among all three groups of patients on both imaging sequences. Additionally, there was no statistical difference in the volume of PVL adjacent to the temporal horns of the lateral ventricles on either sequence. Interestingly, patients with migraine had increased numbers of PVL adjacent to the temporal horn of the lateral ventricles on T2WI $(\mathrm{P}<0.0097)$ compared to RRMS, this trend was not seen on FLAIR. The earliest clinical presentation of RRMS is CIS. There was no significant difference between RRMS and CIS in PVL count or volume for any anatomical region analyzed in Figure 2.

In the next part of the study, we applied binary logistic regression analysis, as described in the methods section, to identify the maximal accuracy and its corresponding threshold for both lesion counts and lesion volume that would differentiate migraine from RRMS and CIS. This was done separately for the count and the volume of lesions adjacent to the posterior horn, the body of the lateral ventricles, total lesion count as well as total lesion volume for both FLAIR and T2WI. The highest accuracy to correctly diagnose CIS vs. migraine was reached when comparing the volume of PVL adjacent to the body of the lateral ventricles, 0.857 , for FLAIR images and for the posterior horn and body of the lateral ventricles, both at 0.833 , for T2WI. The highest accuracy to correctly diagnose RRMS vs. migraine was reached when comparing the volume of PVL adjacent to the body of the lateral ventricles, 0.75 , for FLAIR images. The corresponding volume or count threshold yielding the maximal accuracy to distinguish between RRMS and CIS versus migraine is provided in Supplemental Table 1.

As for the count of PVL, the highest accuracy to correctly diagnose RRMS counting the number of PVL adjacent to the body of lateral ventricles was 0.662 on FLAIR ( 2 or more PVL) and 0.721 on T2WI ( 3 or more PVL). The highest accuracy to correctly diagnose RRMS counting the number of PVL adjacent to the posterior horns of lateral ventricles was 0.662 on FLAIR ( 1 or more PVL) and 0.603 on T2WI (2 or more PVL). In all cases, counting the total number of PVL adjacent to all four anatomical areas of PVL provided equal or inferior accuracy as compared to analyzing PVL adjacent to the body of the lateral ventricles.

In the last part of our study, we compared PVL in patients with RRMS and ischemic stroke. All our stroke patients were between 37 and 58 years old. The mean age of patients with stroke (51.1 years) was significantly higher as compared to patients with CIS (34.8 years) or RRMS (39.4 years) (Table 1). Thus, from the total group of 36 RRMS patients we excluded patients younger than 37 years of age. The final RRMS group consisted of 19 patients, as depicted in Table 3 . The mean ages $( \pm$ SEM) of "age-matched" RRMS and stroke were $46 \pm 7.2$ and $51.1 \pm 6.9$, respectively.

As shown on Table 4, there were no significant differences in the total number and volume of PVL in ischemic stroke patients as compared to the age-matched RRMS patients.

Additionally, there were no significant findings for any of the anatomical areas regarding both lesion count and volume; this was consistent in both T2WI and FLAIR (Supplemental Table 2).

\section{Discussion}

The recent MS criteria $[2,3,15]$ incorporate the presence of PVL on brain MRI for MS diagnosis among young subjects with CIS. However, the specificity of PVL for MS among patients with neurological diseases has not been systematically addressed [3]. FLAIR provides increased sensitivity of demyelinating lesion detection especially in the periventricular location as it can differentiate the signal from the CSF, in the lateral ventricles, from the lesion itself [16-18]. Another important issue is the presence of nonspecific T2 hyperintensities adjacent to the frontal horns of the lateral ventricles in healthy subjects, so-called frontal capping, which can be mistakenly counted as PVL [12].

Our results strongly suggest that PVL adjacent to the anterior and temporal horns of the lateral ventricles are not specific for patients with CIS/RRMS as compared to patients with migraine. In contrast, the volume of PVL adjacent to the posterior horn and body of the lateral ventricles was significantly increased in both RRMS and CIS using different MRI modalities (e.g., FLAIR and T2WI). As related to PVL count, only the FLAIR modality showed significantly increased count of PVL adjacent to both the posterior horn and the body of the lateral ventricles in RRMS. The PVL count was not significantly different between CIS and migraine in any anatomical region.

Based on the binary logistic regression analysis, the highest accuracy in distinguishing between RRMS and migraine was seen when using volume of PVL adjacent to the body of the lateral ventricles instead of PVL count. However, using PVL volume may not be a practical approach in the clinical setting because volumetric analysis software may not be available. Based on our analysis, a reasonable accuracy in distinguishing between RRMS and migraine could be reached by using the following MRI criteria: one or more PVL adjacent to posterior horns as seen on FLAIR, two or more PVL adjacent to the body of the lateral ventricles as seen on FLAIR or three or more PVL adjacent to the body of the lateral ventricles as seen on T2WI (Supplemental Table 1). The recent recommendation by Montalban et al. and the 2010 revised McDonald MS diagnostic criteria [1,3] have decreased the number of PVL from three to one to prove MS dissemination in space as compared to original Barkhof's criteria and the 2005 revised McDonald MS diagnostic criteria $[19,20]$. This change has significantly simplified criteria for $\mathrm{MD}$ diagnosis but is raising concerns about the specificity of the 2010 criteria among patients with migraine. The prevalence of migraine in woman with MS may reach 29\% [9].

\begin{tabular}{|l|l|l|l|}
\hline Patient Group & $\begin{array}{l}\text { Number of patients } \\
\text { (Male/Female Ratio) }\end{array}$ & Age, Mean \pm SD & $\begin{array}{l}\text { Minimum Age } \text { I } \\
\text { Maximum Age }\end{array}$ \\
\hline RRMS & $19(4 / 15)$ & $46.7 \pm 7.2$ & $37 / 58$ \\
\hline Stroke & $18(11 / 7)$ & $51.1 \pm 6.9$ & $37 / 58$ \\
\hline
\end{tabular}

Table 3: Age and Gender Data for Age-Matched RRMS and Stroke Patient Groups.

\begin{tabular}{|c|c|c|c|}
\hline $\begin{array}{c}\text { Neuroimaging } \\
\text { Parameters }\end{array}$ & $\begin{array}{c}\text { RRMS } \\
(\mathbf{n = 1 9 )}\end{array}$ & $\begin{array}{c}\text { Stroke } \\
(\mathbf{n = 1 8})\end{array}$ & $\begin{array}{c}\text { RRMS vs. Stroke } \\
\text { (P-value }\end{array}$ \\
\hline T2WI & & & \\
\hline Total PVL Volume & $1583 \pm 341.2^{*}$ & $2154 \pm 854.7$ & 0.531 \\
\hline Total PVL Count & $4.895 \pm 0.5402$ & $4.444 \pm 0.5437$ & 0.5608 \\
\hline FLAIR & & & \\
\hline Total PVL Volume & $3831 \pm 912.8$ & $5311 \pm 1956$ & 0.49 \\
\hline Total PVL Count & $6.842 \pm 0.5936$ & $7.333 \pm 0.4501$ & 0.5174 \\
\hline
\end{tabular}

* Values are the Mean \pm SEM

* Unpaired t-test was used for statistical analysis and calculation of p-value.

Table 4: PVL Volume and Count in Age-Matched RR-MS and Stroke Patients. 
Citation: Casini G, Yurashevich M, Vanga R, Dash S, Dhib-Jalbut S, et al. (2013) Are Periventricular Lesions Specific for Multiple Sclerosis? J Neurol Neurophysiol 4: 150. doi:10.4172/2155-9562.1000150

We and others have reported that acute demyelinating lesions in patients with RRMS may have restricted diffusion and may be nonenhancing on brain MRI similar to patients with acute stroke [21,22]. Comparison of age-matched ischemic stroke and RRMS patients provided no significant differences in both volume and count of PVL adjacent to any of the four anatomic regions and in total PVL volume and count (Table 4). To our knowledge, this finding has not been previously described. The middle-aged patients presenting with acute symptomatic periventricular lesion may represent a diagnostic challenge in clinical practice.

\section{Acknowledgement}

The authors thank Joan Moore for technical assistance in manuscript preparation.

\section{Funding}

G.C. research activity was supported in part by the Research Scholarship Award from the Foundation of the Consortium of Multiple Sclerosis Centers.

K.E.B. research activity was supported in part by NIH grant K23NS052553

\section{References}

1. Polman $\mathrm{CH}$, Reingold SC, Banwell B, Clanet M, Cohen JA, et al. (2011) Diagnostic criteria for multiple sclerosis: 2010 revisions to the McDonald criteria. Ann Neurol 69: 292-302.

2. Swanton JK, Fernando K, Dalton CM, Miszkiel KA, Thompson AJ, et al. (2006) Modification of MRI criteria for multiple sclerosis in patients with clinically isolated syndromes. J Neurol Neurosurg Psychiatry 77: 830-833.

3. Montalban X, Tintoré M, Swanton J, Barkhof F, Fazekas F, et al. (2010) MRI criteria for MS in patients with clinically isolated syndromes. Neurology 74: 427434.

4. de Lau LM, Smith AD, Refsum H, Johnston C, Breteler MM (2009) Plasma vitamin B12 status and cerebral white-matter lesions. J Neurol Neurosurg Psychiatry 80: 149-157.

5. Scher Al, Gudmundsson LS, Sigurdsson S, Ghambaryan A, Aspelund T, et al. (2009) Migraine headache in middle age and late-life brain infarcts. JAMA 301: 2563-2570.

6. de Leeuw FE, de Groot JC, Achten E, Oudkerk M, Ramos LM, et al. (2001) Prevalence of cerebral white matter lesions in elderly people: a population based magnetic resonance imaging study. The Rotterdam Scan Study. J Neurol Neurosurg Psychiatry 70: 9-14.

7. D'Amico D, La Mantia L, Rigamonti A, Usai S, Mascoli N, et al. (2004) Prevalence of primary headaches in people with multiple sclerosis. Cephalalgia 24: 980-984.

8. Putzki N, Pfriem A, Limmroth V, Yaldizli O, Tettenborn B, et al. (2009)
Prevalence of migraine, tension-type headache and trigeminal neuralgia in multiple sclerosis. Eur J Neurol 16: 262-267.

9. Kister I, Munger KL, Herbert J, Ascherio A (2012) Increased risk of multiple sclerosis among women with migraine in the Nurses' Health Study II. Mult Scler 18: $90-97$.

10. Jacobs LD, Beck RW, Simon JH, Kinkel RP, Brownscheidle CM, et al. (2000) Intramuscular interferon beta-1a therapy initiated during a first demyelinating event in multiple sclerosis. CHAMPS Study Group. N Engl J Med 343: 898-904.

11. Poser CM, Paty DW, Scheinberg L, McDonald WI, Davis FA, et al. (1983) New diagnostic criteria for multiple sclerosis: guidelines for research protocols. Ann Neurol 13: 227-231.

12. Filippi M, Gawne-Cain ML, Gasperini C, vanWaesberghe JH, Grimaud J et al. (1998) Effect of training and different measurement strategies on the reproducibility of brain MRI lesion load measurements in multiple sclerosis. Neurology 50: 238-244.

13. Zou KH, O'Malley AJ, Mauri L (2007) Receiver-operating characteristic analysis for evaluating diagnostic tests and predictive models. Circulation 115: 654-657.

14. Bender R, Grouven U (1998) Using binary logistic regression models for ordinal data with non-proportional odds. J Clin Epidemiol 51: 809-816.

15. Lo CP, Kao HW, Chen SY, Hsueh CJ, Lin WC, et al. (2009). Prediction of conversion from clinically isolated syndrome to clinically definite multiple sclerosis according to baseline MRI findings: comparison of revised McDonald criteria and Swanton modified criteria. J Neurol Neurosurg Psychiatry 80: 1107-1109.

16. Tubridy N, Barker GJ, Macmanus DG, Moseley IF, Miller DH (1998) Threedimensional fast fluid attenuated inversion recovery (3D fast FLAIR): a new MRI sequence which increases the detectable cerebral lesion load in multiple sclerosis. Br J Radiol 71: 840-845.

17. Barkhof $F$, Scheltens $P(2002)$ Imaging of white matter lesions. Cerebrovasc Dis 13 Suppl 2: 21-30.

18. Herskovits EH, Itoh R, Melhem ER (2001) Accuracy for detection of simulated lesions: comparison of fluid-attenuated inversion-recovery, proton densityweighted, and T2-weighted synthetic brain MR imaging. AJR Am J Roentgeno 176: $1313-1318$.

19. Barkhof F, Filippi M, Miller DH, Scheltens P, Campi A, et al. (1997) Comparison of MRI criteria at first presentation to predict conversion to clinically definite multiple sclerosis. Brain $120: 2059-2069$.

20. Polman CH, Reingold SC, Edan G, Filippi M, Hartung HP, et al. (2005) Diagnostic criteria for multiple sclerosis: 2005 revisions to the "McDonald Criteria". Ann Neurol 58: 840-846.

21. Balashov KE, Lindzen E (2012) Acute demyelinating lesions with restricted diffusion in multiple sclerosis. Mult Scler 18: 1745-1753.

22. Zecca C, Cereda C, Wetzel S, Tschuor S, Staedler C, et al. (2012) Diffusionweighted imaging in acute demyelinating myelopathy. Neuroradiology 54: 573578. 